\title{
Calculation of reflectance and transmittance of optical birefringent networks based on cholesteric liquid crystals
}

\author{
Dariusz Grzelczyk ${ }^{\mathrm{a}^{*}}$ (D) \\ Jan Awrejcewicz ${ }^{\mathrm{a}}$ iD \\ a Lodz University of Technology, Department of Automation, Biomechanics and Mechatronics, 1/15 Stefanowskiego Str., 90-924 Lodz, \\ POLAND. E-mail: dariusz.grzelczyk@p.lodz.pl, jan.awrejcewicz@p.lodz.pl. \\ *Corresponding author
}

http://dx.doi.org/10.1590/1679-78255000

\begin{abstract}
In this paper we calculated reflectance and transmittance of different optical birefringent networks sandwiched between two isotropic media. To model optical phenomena in the considered systems, we applied the $4 \times 4$ matrix method. Some interesting reflectance and transmittance spectra and polar plots for different system parameters and arbitrary incident monochromatic light were reported. The illustrated and discussed results can be useful for understanding optical phenomena in numerous birefringent media. Especially, the reflectance and transmittance spectra computed in this article can be suitable for analysis of more advanced contemporary optical systems, i.e. photonic band gap materials based on cholesteric liquid crystals.
\end{abstract}

\section{Keywords}

Photonic crystals, liquid crystals, $4 \times 4$ matrix method, reflectance, transmittance.

\section{INTRODUCTION}

Birefringence is the optical property of materials having refractive indices, which depend both on the polarization and propagation direction of the incident light. The best characterized materials possessing these properties are crystals, because their refractive indices are well defined due to their specific structures. Among the mentioned crystalline structures, special importance have the so-called liquid crystals (LCs). Liquid crystals are the interesting fourth state of matter that lies between the crystalline solid and amorphous liquid states. In some temperature ranges, these materials exhibit both the properties of liquids and the properties of crystalline solid state. Most of them are organic compounds with elongated molecules that influence their physical properties.

The classification of LCs includes three types of liquid crystalline phases, i.e. nematic, smectic, and cholesteric liquid crystals (CLCs). The abovementioned groups differ in physical properties, especially in optical ones. However, due to the strongly periodic helical structure, cholesteric liquid crystals are characterized by unique optical properties. First of them is the abovementioned optical birefringence, i.e. this substance have two different refractive indices. It means that in such an optical media, the propagating wave of the monochromatic light decomposes into two waves, which propagate with different velocities. CLCs also exhibit optical activity and circular dichroism. However, the most popular engineering applications of them are caused by selective reflection of the incident light. The color (wavelength) of the reflected light depends on mechanical stress, temperature, external electric and magnetic fields, etc.

Due to the abovementioned properties, LCs have been used in practical applications for many years, for instance in liquid crystal displays (LCDs). Nowadays, most of phones, computers, television sets and other electronic devices implement LCDs as a one of the most important optical display systems for high-performance flat panel display of information. This is mainly arises from such advantages as flat panel, large area, light weight, high definition, low driving 
voltages and low power consumption (Yeh and Gu, 1999). However, CLCs seem to be also the promising candidates for numerous different photonic applications. This is why, currently, a lot of attention is paid to the photonic crystals (PCs) having a three-dimensional (3D) ordered structure with a periodicity of the optical wavelength, whose optical properties, due to their periodic dielectric structures, are similar to the properties of LCs. In such materials, novel physical concepts have been predicted theoretically and different engineering applications of PCs have been proposed (Ozaki et al., 2007; Cardador et al., 2017; Escobar et al., 2018).

Due to the abovementioned advantages, PCs have attracted much attention from both fundamental studies and practical applications in optical circuits, devices and interconnects (Guo et al., 2010; Notomi, 2010; Prasmusinto et al., 2017). Among the most known new applications of PCs, one can mention light-emitting diodes (Wierer et al., 2009; Wiesmann et al., 2009), optical switches (Belotti et al., 2008; Nozaki et al., 2014), colour filters (Huang and Zhang, 2011; Lin et al., 2015), gas sensors (Hodgkinson and Tatam, 2013; Xu et al., 2013; Cardador et al., 2017) and lasers (Noda, 2010; Hirose et al., 2014; Ortega et al., 2018). Recently, photonic crystals find applications in building all-optical computers and communication systems, using photonic crystal logic gates for faster computers and mobile devices with high both speed processing of data and transmission rates. An interesting literature review in this area can be found in the review paper (Hussein et al., 2018). Also, the most recent articles pay much attention to the theoretical study and new applications of PCs. For instance, Cardador et al. (Cardador et al., 2017) designed and optimized theoretically a macroporous silicon photonic crystal for its use in gas sensing applications and IR optical filters, confirming them as promising candidates for precise optical gas sensors. Tavousi and Heidarzadeh (Tavousi and Heidarzadeh, 2018) realised a high efficiency multichannel drop filter by using a square-lattice-type photonic crystal and a circular ring resonator. Mandal (Mandal, 2018) demonstrated a visible frequency plasmonic perfect absorber made of a thin metal layer with an array of cylindrical grooves used to achieve perfect absorption at tunable wavelengths. Escobar et al. (Escobar et al., 2018) fabricated an active 1D photonic waveguide of porous silicon capable of concentrating light emission, which have potential applications in different integrated optoelectronic devices. In other paper, Zhang et al. (Zhang et al., 2018) proposed a novel compact photonic crystal ring resonator configuration to realise high-efficiency waveguided add-drop filtering.

In general, photonic crystals contain a periodic distribution of both refractive indices in one, two, or three dimensions, and can be used to prohibit, confine and control light propagation in a specific wavelength bandwidth (Lin et al., 2013; Cardador et al., 2017). One of the most important forms of PCs are cholesteric liquid crystals (CLCs) that spontaneously form periodic helical structures (molecules are self-assembled). If the pitch of the CLC helix is of the order of magnitude of the wavelength of the visible light, the material can exhibit photonic properties. In this case, CLCs present a photonic band gap. It means that circularly polarized light with the same handedness as that of the helix cannot propagate in a certain range of frequencies. For instance, the photonic character of CLCs is the basis for the application of these materials as low-threshold distributed feedback lasers (Ortega et al., 2018).

The mentioned helical structure of PCs leads to selective reflection for circularly polarized light in different optical systems (Morris et al., 2008; Choi et al., 2009; Balamurugan and Liu, 2016; Nemati et al., 2018; Ortega et al., 2018). The birefringence is typically limited to 0.3-0.4 for colourless organic compounds, and therefore the bandwidth is usually less than $100 \mathrm{~nm}$ in the visible spectrum (Balamurugan and Liu, 2016). However, there are also materials with different bandwidths. For instance, Fleming et al. (Fleming et al., 2002) reported measurements and simulations of a 3D tungsten crystal that has a large photonic bandgap at infrared wavelengths (from about 8 to $20 \mu \mathrm{m}$ ). An interesting review of the methods of fabrication of various photonic band gap materials based on cholesteric liquid crystals are presented in one of the recent review papers (Balamurugan and Liu, 2016). Especially, that review paper covers the methodology used recently, when the fabrication of photonic band gap materials arose.

It should be noticed that optical properties of LCs strongly depend on the orientation of the liquid crystal molecules. By applying voltage across the transparent electrodes, an electric field obtained inside the liquid crystal can be used to control the orientation of these molecules. This leads to a change in the property of the considered optical systems (Yeh and Gu, 1999). In turn, the performance of photonic crystal devices depends strongly on their geometry, mechanical stress and defects inside (Hart et al., 2012). An interesting literature review on the important defect types, their origins as well as interactions during the bulk crystal growth from the melt and selected epitaxial processes is given in one of the recent review papers (Rudolph, 2016). Some aspects of the effect of anisotropy on defect mode features in CLCs was investigated by Gevorgyan (Gevorgyan, 2018). Yang et al. (Yang et al., 2015) investigated the effect of stress-induced anisotropy on localised mode in photonic crystal and they found that the change in the crystal thickness, caused by stress, leads to localised modes to move linearly in the transmission spectrum. Also recently, Avendanno and Martinez (Avendanno and Martinez, 2018) investigated the transmission spectra in a 1-dimensional dielectric multilayer photonic structure, which contains a cholesteric liquid crystal elastomer layer as a defect. It should be emphasized that although the issues mentioned here are really interesting, they are not considered in detail in this study. 
In this paper, we focused on a theoretical analysis of optical phenomena in different birefringent media, with particular reference to the structure of the CLCs. In order to calculate the reflectance and the transmittance of the investigeted optical system, we implemented a computer algorithm which realizes the $4 \times 4$ matrix method. This method gives the exact solutions, since it takes into account both the effect of refraction and multiple reflections at the interfaces of the dielectric discontinuities. And although we investigated only the chosen birefringent networks, the implemented algorithm can be adopted to model optical phenomena of other optical systems, for instance other types of photonic crystals with a known distribution of the dielectric structure.

The rest of the paper is organized as follows. Section 2 summarizes the matrix methods applied to birefringent networks, especially the conventional Jones calculus, the extended Jones $2 \times 2$ matrix method and the Berreman $4 \times 4$ matrix method. The investigated optical system and crucial stages of the implemented matrix method are presented in Section 3. Section 4 contains calculations and discussion of the reflectance and the transmittance obtained for different optical systems. The article is concluded in the last Section 5 with a brief summary of the main obtained results.

\section{METHODS - A BRIEF LITERATURE REVIEW}

Optical phenomena and the transmission of light through birefringent optical media (networks) have been treated by different methods. In the conventional Jones calculus, each optical element (wave plate, liquid crystal layer, etc.) is represented by a $2 \times 2$ matrix, and reflection/refraction of the incident light at the plate surfaces (dielectric discontinuities) are neglected. Moreover, this method is limited to normally incident light and does not explain the leakage of off-axis light through a pair of crossed ideal polarizers (Gu and Yeh, 1999). For treating the transmission of off-axis light in a general birefringent optical media, the extended Jones matrix method should be used, which takes into consideration the single reflection at the interfaces. This method is adequate for numerous practical applications, and has been widely used in the analysis of numerous optical birefringent systems. Gu and Yeh (Gu and Yeh, 1999) applied the extended Jones matrix method in the analysis of compensators for liquid crystal displays. MacGregor (MacGregor, 1990) presented a model for computing the conoscopic figures produced by a uniformly oriented liquid crystal in a thin cell observed with a polarizing microscope. Lien (Lien, 1990a; 1990b) used Jones matrix methods for the high pretilt twisted nematic cell and for the twisted nematic liquid-crystal display at oblique incidence. Ong (Ong, 1991a; 1991b) analysed electro-optics of electrically controlled birefringence liquid-crystal and twisted nematic liquid-crystal displays at oblique angle of the incident light. In 2000, $\mathrm{Li}$ ( $\mathrm{Li}, 2000$ ) applied the Jones matrix method to ellipsometry, i.e. for investigating the dielectric properties of thin film. In 2010, Chen and co-investigators (Chen et al., 2010) used a new $2 \times 2$ matrix method and discussed the polarization state of transmitted waves through the cholesteric liquid crystals. Most recently, with the help of the concept of $2 \times 2$ transfer matrix method, Kumar et al. (Kumar et al., 2018) investigated comparison between dielectric-dielectric photonic crystal and birefringent-dielectric structure with and without the gradation in the thickness of the layers of the proposed structure.

On the contrary to the aforementioned methods dedicated to the birefringent networks, the exact solutions can be obtained by using the $4 \times 4$ matrix method, which takes into account both the effect of refraction and multiple reflections between plate interfaces (Gu and Yeh, 1999). This approach has been applied by many researches for different optical systems. For instance, Schwelb (Schwelb, 1986) analysed lossy gyroelectromagnetic layers in polar and longitudinal orientation. Chen et al. (Chen et al., 1996) employed the $4 \times 4$ matrix method to liquid crystal displays. Using the $4 \times 4$ matrix method, Ivanov and Sementsov (Ivanov and Sementsov, 2000) modelled the propagation of electromagnetic waves in stratified bianisotropic chiral structures. Yang et al. (Yang et al., 2015) also used this method to investigate the effect of stress-induced anisotropy on localized mode in photonic crystal. Most recently, the $4 \times 4$ matrix method were also used by Ortega and his co-investigators (Ortega et al., 2018) to study different kinds of cells of cholesteric liquid crystal lasers. Due to abovementioned anisotropic properties of CLCs and PCs, also in this paper we used the exact $4 \times 4$ matrix method, which is shortly presented in Section 3.

\section{MODELLING OF OPTICAL PHENOMENA IN LIQUID CRYSTALS}

\subsection{Model of the considered system}

Figure 1(a) presents the model of the cholesteric liquid crystal embedded in the Cartesian coordinate system $x y z$ and sandwiched between two (i.e. lower and upper) homogenous isotropic media. The entire considered CLC with thickness $d$ is divided into $N$ equal multiple layers parallel to the $x y$ plane. Therefore, each layer can be considered as an anisotropic homegeneous medium. The incident light propagates in the lower medium $(n=0)$, next partially propagates in the liquid crystal $(n=1,2, \ldots, N)$ and the upper isotropic medium $(n=N+1)$. The $c$-axis (optical axis) of the CLC lies in $x y$ 
plane and changes periodically along the $z$-direction. Periodic helical structure of the CLC is characterized by the pitch $p$. Both lower and upper isotropic medium is quantified by a refractive index $n_{s}$. The liquid crystal has two refractive indices, i.e. $n_{o}$ and $n_{e}$, for the ordinary $(o)$ and extraordinary $(e)$ waves, respectively. We assumed that all refractive indices do not depend on the wavelength of the incident light.

Figure 1(b) presents orientation of the wave vector $\mathbf{k}$ of the incident non-polarized monochromatic light with wavelength $\lambda$, in the lower isotropic medium. The angle $\theta$ is measured from the $z$-axis to the direction of the wave wector $\mathbf{k}$, while the angle $\phi$ is measured from $x$-axis to the projection of the $\mathbf{k}$ director on the $x y$ plane.

(a)

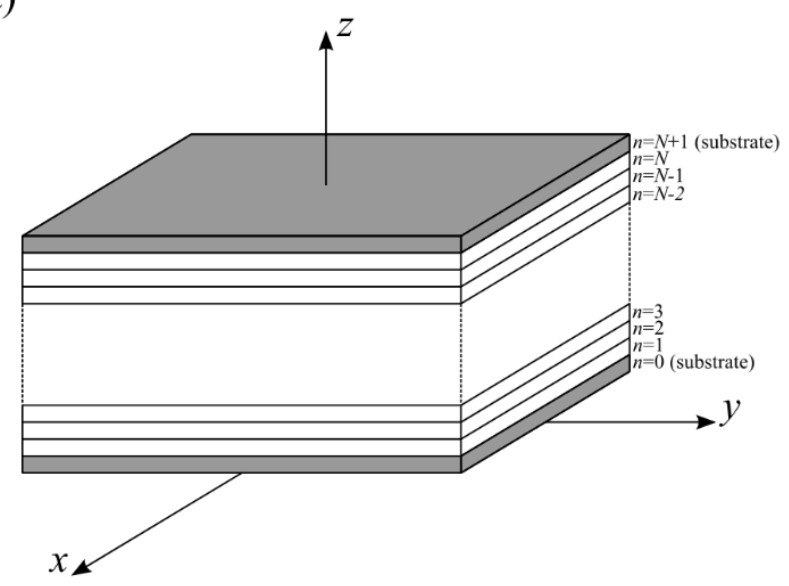

(b)

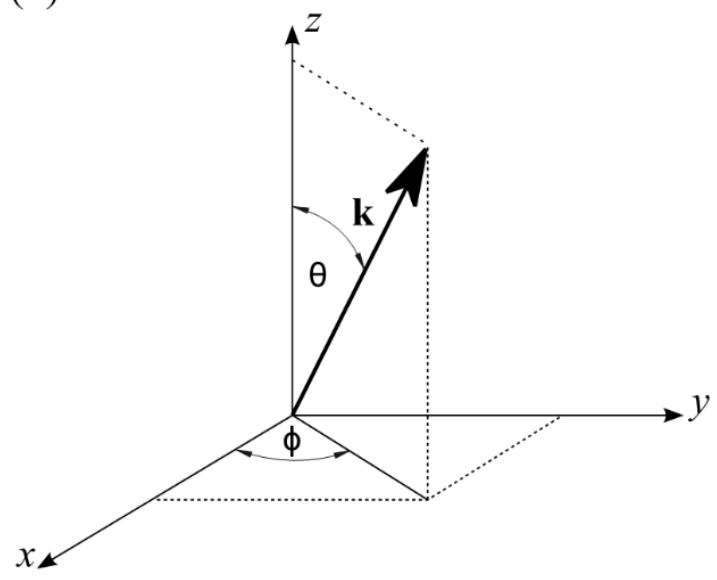

Figure 1: Model of the investigated optical network sandwiched within two isotropic media (a) and orientation of the wave vector $\mathbf{k}$ of the arbitrarily incident light (b).

\subsection{The $4 \times 4$ matrix method}

In this study, we adopted the $4 \times 4$ matrix method on the basis of mathematical formalism presented in monograph (Yeh and $\mathrm{Gu}, 1999$ ). Therefore, only the main and crucial stages of this method are presented below.

In this method, the dielectric tensors $\hat{\boldsymbol{\varepsilon}}(n)$ for the individual liquid crystal layers $(n=1,2, \ldots, N)$ and bounding substrates ( $n=0$ and $n=N+1$ ) are determined first. For both substrates

$\hat{\boldsymbol{\varepsilon}}(0)=\hat{\boldsymbol{\varepsilon}}(N+1)=\varepsilon_{0}\left(\begin{array}{ccc}n_{s}^{2} & 0 & 0 \\ 0 & n_{s}^{2} & 0 \\ 0 & 0 & n_{s}^{2}\end{array}\right)$

where $\varepsilon_{0}=8.85 \cdot 10^{-12} \mathrm{~F} / \mathrm{m}$ is the vacuum permittivity. In case of uniaxial liquid crystal medium, in each $n$-th layer, the orientation of the optical axes $\mathbf{c}(n)$ of the molecules in the laboratory coordinate system can be described by two angles, i.e. $\theta_{c}(n)$ and $\phi_{c}(n)$. The angle $\theta_{c}(n)$ (tilt angle) is measured from the $z$-axis to the direction of the vector $\mathbf{c}(n)$, while the angle $\phi_{c}(n)$ (twist angle) is measured for $x$-axis to the projection of the $\mathbf{c}(n)$ director on the $x y$ plane. As a result, in each $n$-th layer, the vector $\mathbf{c}(n)(n=1,2, \ldots, N)$ has the following form

$\mathbf{c}(n)=\sin \theta_{c}(n) \cos \phi_{c}(n) \cdot \mathbf{x}+\sin \theta_{c}(n) \sin \phi_{c}(n) \cdot \mathbf{y}+\cos \theta_{c}(n) \cdot \mathbf{z}$,

where $\mathbf{x}, \mathbf{y}$ and $\mathbf{z}$ are the unit vectors of the laboratory coordinate system. For an arbitrary orientation of the optical axis $\mathbf{c}(n)$, the dielectric tensor $\hat{\boldsymbol{\varepsilon}}(n)$ can be calculated as

$\hat{\boldsymbol{\varepsilon}}(n)=\varepsilon_{0}\left(\begin{array}{lll}\varepsilon_{x x}(n) & \varepsilon_{x y}(n) & \varepsilon_{x z}(n) \\ \varepsilon_{y x}(n) & \varepsilon_{y y}(n) & \varepsilon_{y z}(n) \\ \varepsilon_{z x}(n) & \varepsilon_{z y}(n) & \varepsilon_{z z}(n)\end{array}\right)$, 
where the elements of the dielectric tensor $\hat{\boldsymbol{\varepsilon}}(n)$ are

$\varepsilon_{x x}(n)=n_{o}^{2}+\left(n_{e}^{2}-n_{o}^{2}\right) \sin ^{2} \theta_{c}(n) \cos ^{2} \phi_{c}(n)$,

$\varepsilon_{x y}(n)=\varepsilon_{y x}(n)=\left(n_{e}^{2}-n_{o}^{2}\right) \sin ^{2} \theta_{c}(n) \sin \phi_{c}(n) \cos \phi_{c}(n)$,

$\varepsilon_{x z}(n)=\varepsilon_{z x}(n)=\left(n_{e}^{2}-n_{o}^{2}\right) \sin \theta_{c}(n) \cos \theta_{c}(n) \cos \phi_{c}(n)$,

$\varepsilon_{y y}(n)=n_{o}^{2}+\left(n_{e}^{2}-n_{o}^{2}\right) \sin ^{2} \theta_{c}(n) \sin ^{2} \phi_{c}(n)$,

$\varepsilon_{y z}(n)=\varepsilon_{z y}(n)=\left(n_{e}^{2}-n_{o}^{2}\right) \sin \theta_{c}(n) \cos \theta_{c}(n) \sin \phi_{c}(n)$,

$\varepsilon_{z z}(n)=n_{o}^{2}+\left(n_{e}^{2}-n_{o}^{2}\right) \cos ^{2} \theta_{c}(n)$.

In the second step, the wave vectors $\mathbf{k}_{\sigma}(n)=\left(\alpha_{\sigma}(n), \beta_{\sigma}(n), \gamma_{\sigma}(n)\right)(\sigma=1,2,3,4)$ of all four elementary waves in all layers $(n=0,1,2, \ldots, N+1)$ are calculated. Let $\mathbf{k}_{1}(0)$ and $\mathbf{k}_{3}(0)$ be the $s$ and $p$ wave vectors of the incoming waves propagating forward, respectively, whereas $\mathbf{k}_{2}(0)$ and $\mathbf{k}_{4}(0)$ denote $s$ and $p$ wave wectors of the reflected waves propagating backward, respectively, in the lower substrate. Moreover, $\mathbf{k}_{1}(N+1)$ and $\mathbf{k}_{3}(N+1)$ denote $s$ and $p$ wave vectors transmitted to the upper substrate, respectively, whereas $\mathbf{k}_{2}(N+1)$ and $\mathbf{k}_{4}(N+1)$ are $s$ and $p$ wave vectors reflected in the upper substrate (their amplitudes are equals zero). In an anisotropic medium $(n=1,2, \ldots, N), \mathbf{k}_{1}(n)$ and $\mathbf{k}_{3}(n)$ denote $o$ and $e$ wave vectors of the transmitted waves, respectively, whereas $\mathbf{k}_{2}(n)$ and $\mathbf{k}_{4}(n)$ denote $o$ and $e$ wave vectors of the reflected waves, respectively. Since the whole layered birefringent medium is homogeneous in the $x y$ plane, $\alpha$ and $\beta$ remain the same throughout the whole layered medium. The components of the wave vectors in both $x$ - and $y$-direction of all elementary waves can be obtained as follow

$\alpha_{\sigma}(n)=n_{s} k_{0} \sin \theta \cos \phi$,

$\beta_{\sigma}(n)=n_{s} k_{0} \sin \theta \sin \phi$,

where $n=0,1,2, \ldots, N, N+1, \sigma=1,2,3,4$ and $k_{0}=2 \pi / \lambda_{0}$ ( $\lambda_{0}$ is the wavelength of the monochromatic light in vacuum). The $z$ components of the vectors $\mathbf{k}_{\sigma}(0)$ and $\mathbf{k}_{\sigma}(N+1)$ are computed as

$$
\begin{aligned}
& \gamma_{1}(0)=\gamma_{3}(0)=n_{s} k_{0} \cos \theta, \\
& \gamma_{2}(0)=\gamma_{4}(0)=-n_{s} k_{0} \cos \theta, \\
& \gamma_{1}(N+1)=\gamma_{3}(N+1)=n_{s} k_{0} \cos \theta, \\
& \gamma_{2}(N+1)=\gamma_{4}(N+1)=-n_{s} k_{0} \cos \theta .
\end{aligned}
$$

Given $\alpha_{\sigma}(n)$ and $\beta_{\sigma}(n)$, the $z$ components $\gamma_{\sigma}(n)$ are determined directly from the wave equation in momentum space

$\mathbf{k} \times(\mathbf{k} \times \mathbf{E})+\omega^{2} \mu \varepsilon \mathbf{E}=\mathbf{0}$, 
where $\mathbf{k}=(\alpha, \beta, \gamma), \mathbf{E}=\left(E_{x}, E_{y}, E_{z}\right)$ is the vector of the electric field, $\omega=2 \pi c / \lambda$ ( $c$ - the speed of light in vacuum), whereas $\mu$ is the magnetic permeability $\mu=\mu_{0}=4 \pi \cdot 10^{-7} \mathrm{H} / \mathrm{m}$ (here the considered optical medium is a diamagnetic substance). The above presented wave equation (7) has the following scalar form

$$
\left(\begin{array}{ccc}
\omega^{2} \mu \varepsilon_{x x}-\beta^{2}-\gamma^{2} & \omega^{2} \mu \varepsilon_{x y}+\alpha \beta & \omega^{2} \mu \varepsilon_{x z}+\alpha \gamma \\
\omega^{2} \mu \varepsilon_{y x}+\alpha \beta & \omega^{2} \mu \varepsilon_{y y}-\alpha^{2}-\gamma^{2} & \omega^{2} \mu \varepsilon_{y z}+\beta \gamma \\
\omega^{2} \mu \varepsilon_{z x}+\alpha \gamma & \omega^{2} \mu \varepsilon_{z y}+\beta \gamma & \omega^{2} \mu \varepsilon_{z z}-\alpha^{2}-\beta^{2}
\end{array}\right) \cdot\left(\begin{array}{l}
E_{x} \\
E_{y} \\
E_{z}
\end{array}\right)=\left(\begin{array}{l}
0 \\
0 \\
0
\end{array}\right) .
$$

In order to obtain non-trivial plane-wave solutions, the determinant of the $3 \times 3$ matrix must vanish in equation (8). This condition gives an equation in $\gamma$ that yields four roots $\gamma_{1}(n), \gamma_{2}(n), \gamma_{3}(n)$ and $\gamma_{4}(n)$ in each $n$-th layer. This equation can be solved analitycally. The roots can be either real or complex. Because all the coefficients of this equation are real, complex roots are always in conjugate pairs. As a result, there exist four modes of propagation within each $n$-th homogeneous layer. Two of them (with positive roots $\gamma_{1}(n)$ and $\left.\gamma_{3}(n)\right)$ propagate forward and the other two (with negative roots $\gamma_{2}(n)$ and $\left.\gamma_{4}(n)\right)$ propagate backward. Two of these roots have always the opposite sign, but the same absolute values. These roots correspond to the ordinary waves, i.e. the wave with positive root propagates forward, whereas the wave with a negative root propagates backward. Two other roots (with different sign and, in general, different absolute values) correspond to the extraordinary waves. Here also the wave with a positive root propagates forward, whereas the wave with negative root propagates backward.

The third stage of the implemented method is devoted to computing the optical polarization vectors $\mathbf{p}_{\sigma}(n)$ of individual elementary waves (with wave vectors $\mathbf{k}_{\sigma}(n)$ ) representing the directions of the electric field as well as the corresponding vectors $\mathbf{q}_{\sigma}(n)$ representing the directions of the magnetic field. For each isotropic media, these vectors, for $s$ and $p$ waves, can be obtained as follow

$\mathbf{p}_{1}(0)=\frac{\mathbf{k}_{1}(0) \times \mathbf{z}}{\left|\mathbf{k}_{1}(0) \times \mathbf{z}\right|}, \mathbf{p}_{2}(0)=\frac{\mathbf{k}_{2}(0) \times \mathbf{z}}{\left|\mathbf{k}_{2}(0) \times \mathbf{z}\right|}, \mathbf{p}_{3}(0)=\frac{\mathbf{k}_{3}(0) \times \mathbf{p}_{1}(0)}{\left|\mathbf{k}_{3}(0) \times \mathbf{p}_{1}(0)\right|}, \mathbf{p}_{4}(0)=\frac{\mathbf{k}_{4}(0) \times \mathbf{p}_{2}(0)}{\left|\mathbf{k}_{4}(0) \times \mathbf{p}_{2}(0)\right|}$,

and

$\mathbf{p}_{1}(N+1)=\frac{\mathbf{k}_{1}(N+1) \times \mathbf{z}}{\left|\mathbf{k}_{1}(N+1) \times \mathbf{z}\right|}, \mathbf{p}_{2}(N+1)=\frac{\mathbf{k}_{2}(N+1) \times \mathbf{z}}{\left|\mathbf{k}_{2}(N+1) \times \mathbf{z}\right|}$,

$\mathbf{p}_{3}(N+1)=\frac{\mathbf{k}_{3}(N+1) \times \mathbf{p}_{1}(N+1)}{\left|\mathbf{k}_{3}(N+1) \times \mathbf{p}_{1}(N+1)\right|}, \mathbf{p}_{4}(N+1)=\frac{\mathbf{k}_{4}(N+1) \times \mathbf{p}_{2}(N+1)}{\left|\mathbf{k}_{4}(N+1) \times \mathbf{p}_{2}(N+1)\right|}$.

In each $n$-th layer of the birefringent medium, these vectors, for $o$ and $e$ waves, have the following forms

$$
\mathbf{p}_{1}(n)=\frac{\mathbf{k}_{1}(n) \times \mathbf{c}(n)}{\left|\mathbf{k}_{1}(n) \times \mathbf{c}(n)\right|}, \mathbf{p}_{2}(n)=\frac{\mathbf{k}_{2}(n) \times \mathbf{c}(n)}{\left|\mathbf{k}_{2}(n) \times \mathbf{c}(n)\right|}, \mathbf{p}_{3}(n)=\frac{\mathbf{p}_{1}(n) \times \mathbf{k}_{3}(n)}{\left|\mathbf{p}_{1}(n) \times \mathbf{k}_{3}(n)\right|}, \mathbf{p}_{4}(n)=\frac{\mathbf{p}_{2}(n) \times \mathbf{k}_{4}(n)}{\left|\mathbf{p}_{2}(n) \times \mathbf{k}_{4}(n)\right|} .
$$

The vectors $\mathbf{q}_{\sigma}(n)$, which correspond to the directions of the magnetic field $\mathbf{H}$ of all elementary waves ( $\sigma=1,2,3,4$ ) propagating in the layered anisotropic optical medium can be expressed as

$\mathbf{q}_{\sigma}(n)=\frac{\mathbf{k}_{\sigma}(n)}{\omega \mu} \times \mathbf{p}_{\sigma}(n)$

In the fourth stage of the method, transition matrices between individual layers are calculated, based on the assumption of continuity of the tangential components of the vectors $\mathbf{p}_{\sigma}(n)$ and $\mathbf{q}_{\sigma}(n)$ at the dielectric interfaces. In the applied approach, we expressed the amplitudes $A_{\sigma}(0)$ of all elementary waves in the lower substrate as a function of the 
amplitudes $A_{\sigma}(N+1)$ of all elementray waves in the upper substrate. This relation is given by the transmittion matrix $M_{4 \times 4}$. With this assumption, the electric field $\mathbf{E}$ in the $z$ coordinate can be expressed as the sum of the electric fields of all elementary waves, in the form

$\mathbf{E}=\sum_{\sigma=1}^{4} A_{\sigma}(n) \mathbf{p}_{\sigma}(n) \exp \left\{i\left[\omega t-\alpha_{\sigma}(n) x-\beta_{\sigma}(n) y-\gamma_{\sigma}(n)\left(z-z_{n}\right)\right]\right\}$,

where $A_{\sigma}(n)$ denote the amplitudes of all elementary waves. Similarly, the magnetic field $\mathbf{H}$ can be expressed as a sum of the magnetic fields of all elementary waves, in the form

$\mathbf{H}=\sum_{\sigma=1}^{4} A_{\sigma}(n) \mathbf{q}_{\sigma}(n) \exp \left\{i\left[\omega t-\alpha_{\sigma}(n) x-\beta_{\sigma}(n) y-\gamma_{\sigma}(n)\left(z-z_{n}\right)\right]\right\}$

At the interface $z=z_{n-1}$, both $x$ and $y$ components of electric and and magnetic fields $\left(E_{x}, E_{y}, H_{x}\right.$ and $\left.H_{y}\right)$ must be continuous, and it gives the set of the following four equations

$\sum_{\sigma=1}^{4} A_{\sigma}(n-1) \mathbf{p}_{\sigma}(n-1) \cdot \mathbf{x}=\sum_{\sigma=1}^{4} A_{\sigma}(n) \mathbf{p}_{\sigma}(n) \cdot \mathbf{x} \exp \left(i \gamma_{\sigma}(n) t_{n}\right)$,

$\sum_{\sigma=1}^{4} A_{\sigma}(n-1) \mathbf{p}_{\sigma}(n-1) \cdot \mathbf{y}=\sum_{\sigma=1}^{4} A_{\sigma}(n) \mathbf{p}_{\sigma}(n) \cdot \mathbf{y} \exp \left(i \gamma_{\sigma}(n) t_{n}\right)$,

$\sum_{\sigma=1}^{4} A_{\sigma}(n-1) \mathbf{q}_{\sigma}(n-1) \cdot \mathbf{x}=\sum_{\sigma=1}^{4} A_{\sigma}(n) \mathbf{q}_{\sigma}(n) \cdot \mathbf{x} \exp \left(i \gamma_{\sigma}(n) t_{n}\right)$

$\sum_{\sigma=1}^{4} A_{\sigma}(n-1) \mathbf{q}_{\sigma}(n-1) \cdot \mathbf{y}=\sum_{\sigma=1}^{4} A_{\sigma}(n) \mathbf{q}_{\sigma}(n) \cdot \mathbf{y} \exp \left(i \gamma_{\sigma}(n) t_{n}\right)$,

where $t_{n}=z_{n}-z_{n-1}(n=1,2, \ldots, N)$ is the thickness of $n$-th layer and equals $d / N$, while $t_{N+1} \equiv 0$. Equations (15) have the following scalar form

$\left(\begin{array}{l}A_{1}(n-1) \\ A_{2}(n-1) \\ A_{3}(n-1) \\ A_{4}(n-1)\end{array}\right)=\mathbf{D}^{-1}(n-1) \mathbf{D}(n) \mathbf{P}(n)\left(\begin{array}{l}A_{1}(n) \\ A_{2}(n) \\ A_{3}(n) \\ A_{4}(n)\end{array}\right)$

where

$\mathbf{D}(n)=\left(\begin{array}{llll}\mathbf{x} \cdot \mathbf{p}_{1}(n) & \mathbf{x} \cdot \mathbf{p}_{2}(n) & \mathbf{x} \cdot \mathbf{p}_{3}(n) & \mathbf{x} \cdot \mathbf{p}_{4}(n) \\ \mathbf{y} \cdot \mathbf{p}_{1}(n) & \mathbf{y} \cdot \mathbf{p}_{2}(n) & \mathbf{y} \cdot \mathbf{p}_{3}(n) & \mathbf{y} \cdot \mathbf{p}_{4}(n) \\ \mathbf{x} \cdot \mathbf{q}_{1}(n) & \mathbf{x} \cdot \mathbf{q}_{2}(n) & \mathbf{x} \cdot \mathbf{q}_{3}(n) & \mathbf{x} \cdot \mathbf{q}_{4}(n) \\ \mathbf{y} \cdot \mathbf{q}_{1}(n) & \mathbf{y} \cdot \mathbf{q}_{2}(n) & \mathbf{y} \cdot \mathbf{q}_{3}(n) & \mathbf{y} \cdot \mathbf{q}_{4}(n)\end{array}\right)$,

and 


$$
\mathbf{P}(n)=\left(\begin{array}{cccc}
\exp \left(i \gamma_{1}(n) t_{n}\right) & 0 & 0 & 0 \\
0 & \exp \left(i \gamma_{2}(n) t_{n}\right) & 0 & 0 \\
0 & 0 & \exp \left(i \gamma_{3}(n) t_{n}\right) & 0 \\
0 & 0 & 0 & \exp \left(i \gamma_{4}(n) t_{n}\right)
\end{array}\right)
$$

$\mathbf{D}(n)$ are called the dynamical matrices, while $\mathbf{P}(n)$ are called the propagation matrices. Finally, the general relation between the amplitudes $A_{\sigma}(0)$ and $A_{\sigma}(N+1)$ has the form

$$
\left(\begin{array}{l}
A_{1}(0) \\
A_{2}(0) \\
A_{3}(0) \\
A_{4}(0)
\end{array}\right)=\mathbf{M}_{4 \times 4}\left(\begin{array}{l}
A_{1}(N+1) \\
A_{2}(N+1) \\
A_{3}(N+1) \\
A_{4}(N+1)
\end{array}\right)
$$

where $\mathbf{M}_{4 \times 4}=\mathbf{T}_{0,1} \mathbf{T}_{1,2} \mathbf{T}_{2,3} \ldots \mathbf{T}_{n-1, n} \ldots \mathbf{T}_{N-1, N} \mathbf{T}_{N, N+1}$ and $\mathbf{T}_{n-1, n}=\mathbf{D}^{-1}(n-1) \mathbf{D}(n) \mathbf{P}(n)$.

Let $A_{s}$ and $A_{p}$ denote the amplitudes of the electric field of $s$ and $p$ waves that incident the anisotropic medium from the lower substrate, respectively. Moreover, let $B_{s}$ and $B_{p}$ denote the amplitudes of the reflected $s$ and $p$ waves at the interface $z=0$, respectively, whereas $C_{s}$ and $C_{p}$ are the amplitudes of the $s$ and $p$ waves transmitted to the upper substrate at the interface $z=d$. As it has been already mentioned, the amplitudes of the reflected waves in the upper isotropic medium equal zero. The relation between all mentioned amplitudes can be presented in the vectormatrix form

$$
\left(\begin{array}{l}
A_{s} \\
B_{s} \\
A_{p} \\
B_{p}
\end{array}\right)=\left(\begin{array}{llll}
M_{11} & M_{12} & M_{13} & M_{14} \\
M_{21} & M_{22} & M_{23} & M_{24} \\
M_{31} & M_{32} & M_{33} & M_{34} \\
M_{41} & M_{42} & M_{43} & M_{44}
\end{array}\right)\left(\begin{array}{l}
C_{s} \\
0 \\
C_{p} \\
0
\end{array}\right)
$$

where $M_{i j}(i, j=1,2,3,4)$ are the components of the matrix $\mathbf{M}_{4 \times 4}$. This relation gives the values of the unknown amplitudes $B_{s}, B_{p}$ of the reflected waves and $C_{s}, C_{p}$ of the transmitted waves, as a function of the known amplitudes $A_{s}$ and $A_{p}$ of the incident waves. Moreover, based on the relation (20), we calculated both the reflection and transmission coefficients given by

$$
\begin{aligned}
& r_{s s}=\left(\frac{B_{s}}{A_{s}}\right)_{A_{p}=0}=\frac{M_{21} M_{33}-M_{23} M_{31}}{M_{11} M_{33}-M_{13} M_{31}}, r_{s p}=\left(\frac{B_{p}}{A_{s}}\right)_{A_{p}=0}=\frac{M_{41} M_{33}-M_{43} M_{31}}{M_{11} M_{33}-M_{13} M_{31}}, \\
& r_{p s}=\left(\frac{B_{s}}{A_{p}}\right)_{A_{s}=0}=\frac{M_{11} M_{23}-M_{21} M_{13}}{M_{11} M_{33}-M_{13} M_{31}}, r_{p p}=\left(\frac{B_{p}}{A_{p}}\right)_{A_{s}=0}=\frac{M_{11} M_{43}-M_{41} M_{13}}{M_{11} M_{33}-M_{13} M_{31}}, \\
& t_{s s}=\left(\frac{C_{s}}{A_{s}}\right)_{A_{p}=0}=\frac{M_{33}}{M_{11} M_{33}-M_{13} M_{31}}, t_{s p}=\left(\frac{C_{p}}{A_{s}}\right)_{A_{p}=0}=\frac{-M_{31}}{M_{11} M_{33}-M_{13} M_{31}},
\end{aligned}
$$


$t_{p s}=\left(\frac{C_{s}}{A_{p}}\right)_{A_{s}=0}=\frac{-M_{13}}{M_{11} M_{33}-M_{13} M_{31}}, t_{p p}=\left(\frac{C_{p}}{A_{p}}\right)_{A_{s}=0}=\frac{M_{11}}{M_{11} M_{33}-M_{13} M_{31}}$

Then, equation (20) can be also given in the following form of two matrix equations

$$
\begin{gathered}
\left(\begin{array}{l}
B_{s} \\
B_{p}
\end{array}\right)=\left(\begin{array}{ll}
r_{s s} & r_{p s} \\
r_{s p} & r_{p p}
\end{array}\right)\left(\begin{array}{l}
A_{s} \\
A_{p}
\end{array}\right), \\
\left(\begin{array}{l}
C_{s} \\
C_{p}
\end{array}\right)=\left(\begin{array}{ll}
t_{s s} & t_{p s} \\
t_{s p} & t_{p p}
\end{array}\right)\left(\begin{array}{l}
A_{s} \\
A_{p}
\end{array}\right) .
\end{gathered}
$$

The fraction of the electromagnetic energy reflected is

$$
R=\frac{B_{s}^{2}+B_{p}^{2}}{A_{s}^{2}+A_{p}^{2}}
$$

while the fraction of the electromagnetic energy transmitted is

$$
T=\frac{C_{s}^{2}+C_{p}^{2}}{A_{s}^{2}+A_{p}^{2}}
$$

which depend on the matrix elements $M_{i j}$ and on the polarization state of the incident $s$ and $p$ waves (i.e. $A_{s}$ and $A_{p}$ ).

Usually, we deal with an incident beam of non-polarized light, therefore the incident light can be written

$$
\left(\begin{array}{c}
A_{s} \\
A_{p}
\end{array}\right)=\frac{E_{o}}{\sqrt{2}}\left(\begin{array}{c}
e^{i \alpha_{1}} \\
e^{i \alpha_{2}}
\end{array}\right)
$$

where $E_{0}$ is the intensity of the electric field of the non-polarized light, and $\alpha_{1}$ and $\alpha_{2}$ are random variables in a sense that the time-averaged quantities $\left[\sin \left(\alpha_{1}-\alpha_{2}\right)\right]$ and $\left[\cos \left(\alpha_{1}-\alpha_{2}\right)\right]$ vanish simultaneously. Then, the fraction of the energy reflected is given by

$R=\frac{1}{2}\left(r_{s s}^{2}+r_{p s}^{2}+r_{s p}^{2}+r_{p p}^{2}\right)$

while the fraction of the energy transmitted is given by

$T=\frac{1}{2}\left(t_{s s}^{2}+t_{p s}^{2}+t_{s p}^{2}+t_{p p}^{2}\right)$

\section{RESULTS}

In what follows we illustrated and discussed some calculations of the reflectance $R$ (and transmittance $T$ ) spectra of the normally incident light, the reflectance $R$ vs. the angle $\theta$, as well as colour polar plots in the full range of the angles $\theta$ and $\phi$ of the incident light. In the presented reflectance spectra, the colours visible under the chart correspond to the colours of the light with wavelength presented on the $x$-axis. In turn, in all polar plots presented in this paper, the radial 
position corresponds to the angle $\theta$ (from 0 , in the middle, to the $90^{\circ}$, at the edges of the plotted polar distributions), whereas the circumferential position corresponds to the angle $\phi$ (from 0 to $360^{\circ}$, as it is shown in plotted distributions). In all cases, we used a monochromatic non-polarized light and different values of the parameters of the investigated system. To test the implemented method, we considered the propagation of light within the homogenous isotropic medium and the propagation of light in the homogenous isotropic medium sandwiched between the other isotropic medium first. Next, we investigated the reflection properties of liquid crystals with different textures, i.e. nematic, homeotropic and helical structures (cholesteric liquid crystal). All calculations were carried out for $N=1000$.

\subsection{Propagation of light in a homogenous isotropic medium}

To test the developed algorithm, we considered the case when the light propagates through the homogenous and lossless isotropic optical medium first, i.e. we assumed that $n_{s}=n_{0}=n_{e}$. In this case, for arbitrary values of the parameters $d, \lambda, \theta, \phi$ and $n_{s}=n_{0}=n_{e}$, in each case we obtained $R=0$ and $T=1$. This is why there are no dielectric discontinuities at the interfaces of the imaginary layers in such optical systems. Therefore, there is no electromagnetic energy reflected, i.e. the incident light is completely transmitted through the investigated optical system. Moreover, we tested the propagation of not normally incident light (i.e. for arbitrary angles $\theta$ and $\phi$, which describe the orientation of the wave wector of the incident beam of the light). In these cases, we also obtained $R=0$ and $T=1$.

\subsection{Propagation of light in a homogenous isotropic medium sandwiched between other isotropic medium}

In this subsection we considered the propagation of light within an isotropic medium sandwiched between other isotropic medium (see parameters of the system in Tab. 1).

Table 1: Parameters of the investigated two different isotropic optical media.

\begin{tabular}{cccc}
\hline \multicolumn{4}{l}{ Isotropic medium sandwiched between other isotropic medium $\left(n_{o}=n_{e} \neq n_{s}\right)$} \\
\hline Quantity & $n_{o}$ & $n_{e}$ & $n_{s}$ \\
Unit & - & - & - \\
Value & 1.55 & 1.55 & 1.3
\end{tabular}

Figure 2 presents reflectance spectra of the investigated system for $d=10 \mu \mathrm{m}$ and different values of the refractive index $n_{s}$ of the substrates. It can be clearly seen that we obtained $R+T=1$ in each case, and therefore usually only reflectance spectra are presented in the further analysis. The obtained results show that the reflection coefficient $R$ is relatively low, and oscillates locally between the minimum and maximum values. This amplitude is greater when the difference between refraction indices is greater. In the presented cases, we have the situation similar to the interferences which occur in Fabry-Perot resonator, whereas the role of the optical mirrors is played by the dielectric interfaces at the surfaces of contacting optical media with different refraction indices.

Figure 3 presents reflectance spectra of the system for different values of the thickness $d$ of the isoptropic medium. In all cases, the maximum value of the amplitude of oscillations is the same $(R \approx 0.03)$, since the difference between the refractive indices of both isotropic media are the same in all presented cases. However, it is clearly visible that for larger values of the thickness $d$, the reflection coefficient $R$ oscillates with a higher frequency.

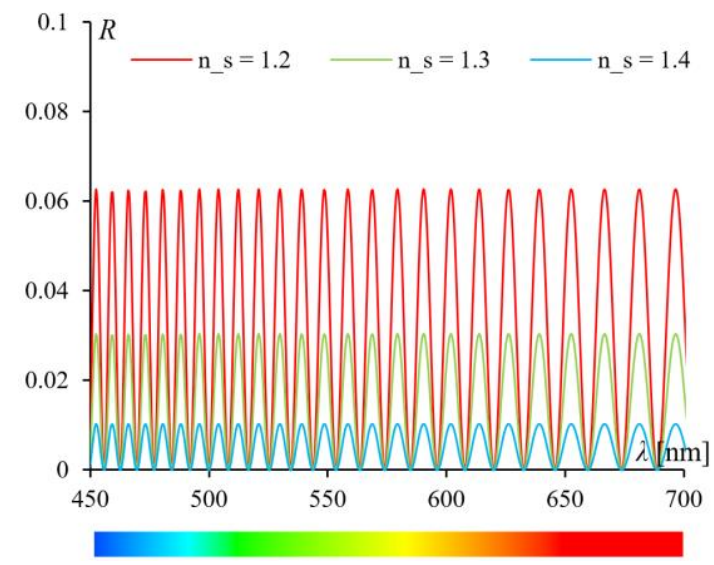

Figure 2: Reflectance spectra for $d=10 \mu \mathrm{m}$ and different values of the refractive index $n_{s}$. 


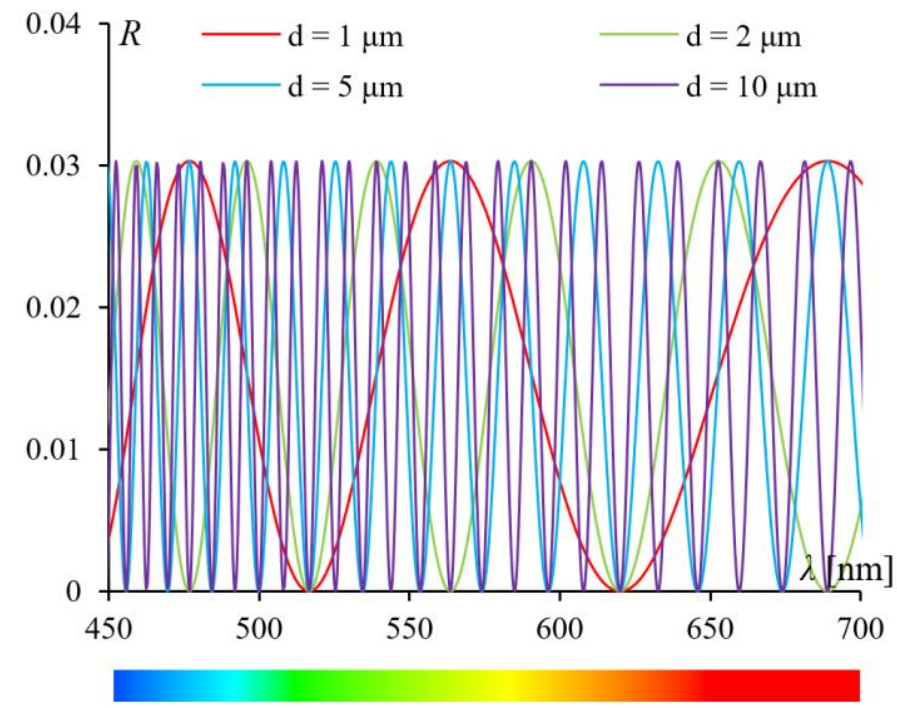

Figure 3: Reflectance spectra for different values of the thickness $d$.

Figure 4 presents the reflectance $R$ vs. the angle $\theta$ for different values of the thickness $d$ and $\lambda=560 \mathrm{~nm}$. For normally incident light, the reflectance is about $R \approx 0.03$, whereas it increases when the angle $\theta$ increases, finally reaching 1 for $\theta \rightarrow 90^{\circ}$. Oscillations of the $R$ coefficient are larger for larger values of the parameter $d$, which can also be seen in the polar plots shown in Fig 5 . Fig. 5 also shows that the reflectance $R$ strongly depends on the angle $\theta$, while it is not dependent on the angle $\phi$ (due to isotropy of the considered optical medium).

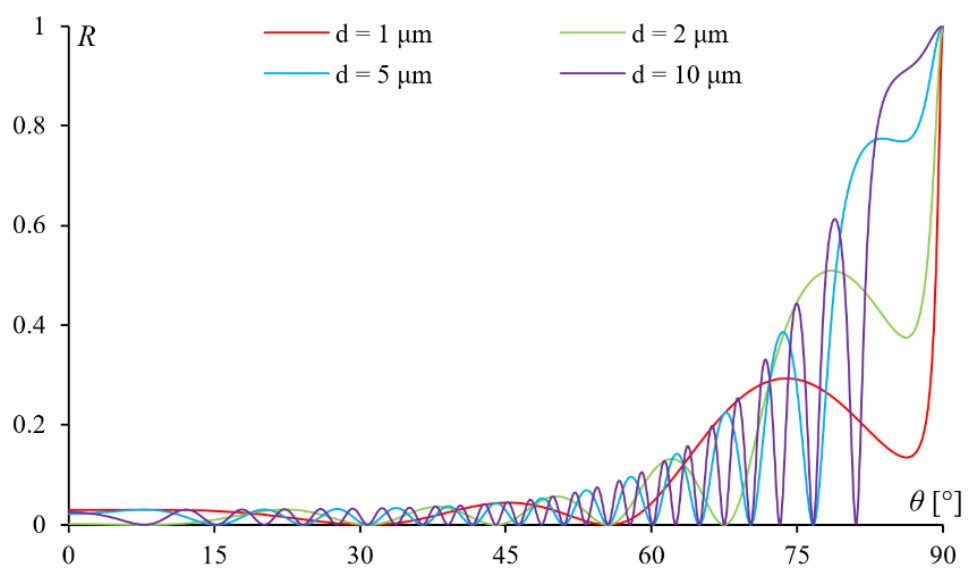

Figure 4: Reflectance vs. the angle $\theta$ for different values of the thickness $d$.

(a)

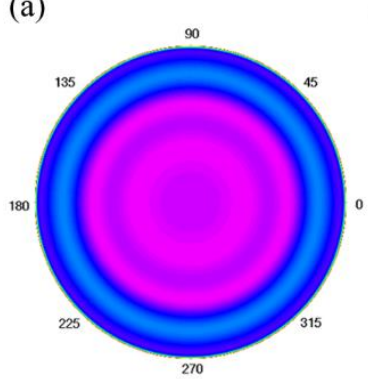

(b)

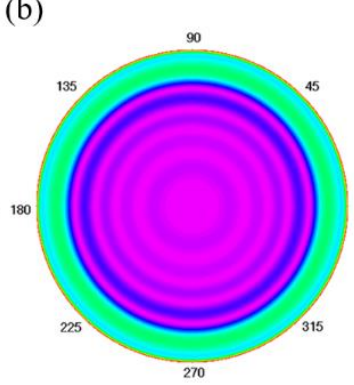

(c)

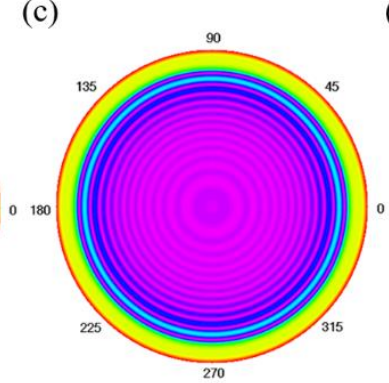

(d)

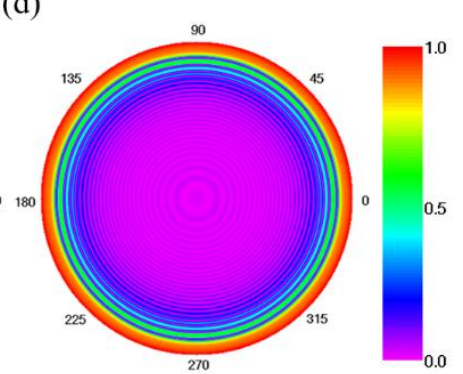

Figure 5: Polar plots of reflectance for different values of $d$ : (a) $d=1 \mu \mathrm{m}$; (b) $d=2 \mu \mathrm{m}$; (c) $d=5 \mu \mathrm{m}$; (d) $d=10 \mu \mathrm{m}$.

\subsection{Propagation of light in the planar structure of LC}

In this subsection we considered planar texture of the liquid crystal sandwiched between two isotropic media. Parameters of the considered system are shown in Tab. 2. 
Table 2: Parameters of the investigated planar structure of $L C$ and isotropic optical medium.

\begin{tabular}{cccc}
\hline Planar structure of $\mathbf{L C}\left(\phi_{c}(n)=0, \theta_{c}(n)=90^{\circ}, n=1,2, \ldots, N\right)$ \\
\hline Quantity & $n_{o}$ & $n_{e}$ & $n_{s}$ \\
Unit & - & - & - \\
Value & 1.55 & 1.65 & 1.3
\end{tabular}

Figure 6 presents reflectance spectra of the optical system for different values of the thickness $d$ of the LC cell. The reflection coefficient oscillates locally between the minimum and the maximum, with different amplitude, depending on the wavelength of the incident light. These oscillations are larger for larger values of the thickness $d$. This regularity is also visible on the polar plots obtained for $\lambda=560 \mathrm{~nm}$ and depicted in Fig. 7. It should be noted that in this case, due to inhomegenous structure of the considered medium, the reflection coefficient $R$, visible in polar plots depicted in Fig. 7 , strongly depends both on the angle $\theta$ and the angle $\phi$. Due to the symmetry of the tensor of the electrical permeability along the $x$ and $y$ axes of the laboratory coordinate system, also the presented distributions of the reflectance (polar plots) are symmetrical with respect to these axes of the coordinate system.

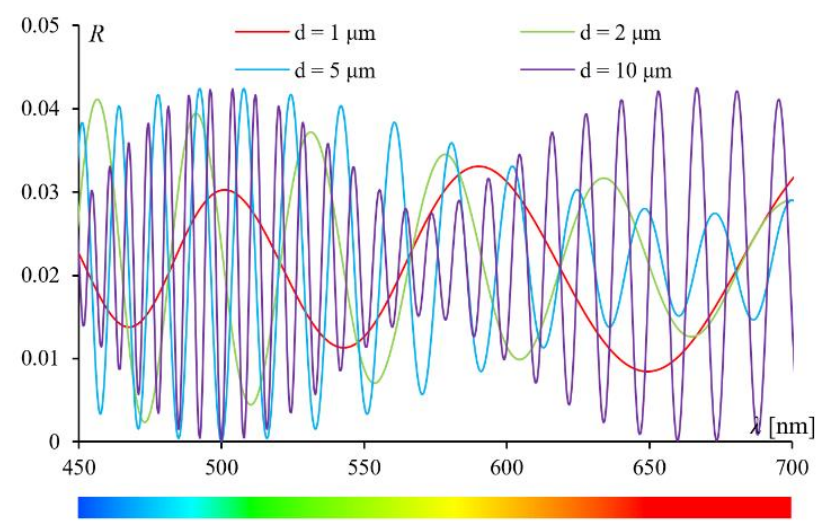

Figure 6: Reflectance spectra for different values of the thickness $d$.

(a)

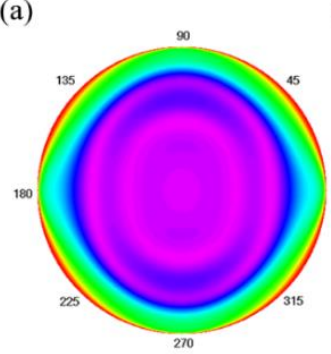

(b)

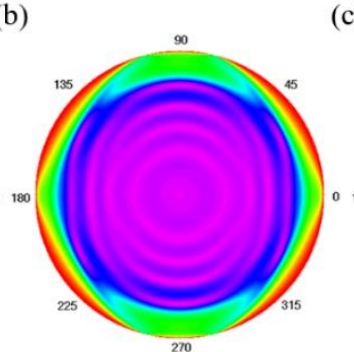

(c)

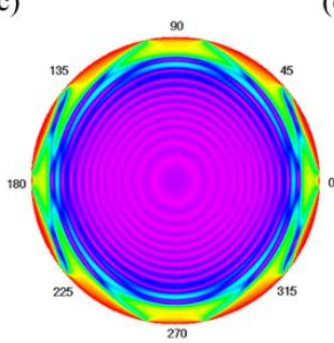

(d)

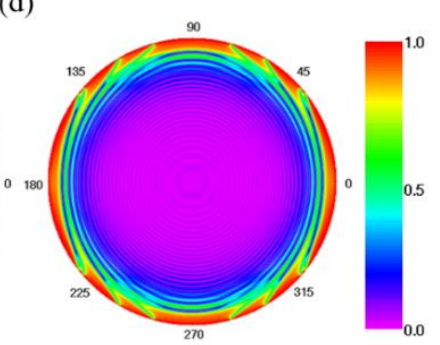

Figure 7: Polar plots of reflectance for different values of $d$ : (a) $d=1 \mu \mathrm{m}$; (b) $d=2 \mu \mathrm{m}$; (c) $d=5 \mu \mathrm{m}$; (d) $d=10 \mu \mathrm{m}$.

\subsection{Propagation of light in the homeotropic structure of LC}

Figure 8 shows the reflectance spectra of the homeotropic texture of the LC (see parameters in Tab. 3) for different values of the thickness $d$. In this case, the optical $c$-axis of liquid crystal is parallel to the $z$-axis. Therefore, the reflection coefficient $R$ oscillates in the same range, which depends only on the refractive indices of the system. Also in this case the frequency of oscillations of the $R$ coefficient is larger for larger values of the thickness $d$. Due to the abovementioned symmetry of the investigated LC, polar plots obtained for $\lambda=560 \mathrm{~nm}$ and visible in Fig. 9 are symmetrical with respect to the $z$-axis, i.e. the reflectance strongly depends on the angle $\theta$, while it does not depend on the angle $\phi$.

Table 3: Parameters of the investigated homeotropic structure of LC and isotropic optical medium.

\begin{tabular}{cccc}
\hline \multicolumn{4}{l}{ Homeotropic structure of $\mathbf{L C}\left(\theta_{c}(n)=0, n=1,2, \ldots, N\right)$} \\
\hline Quantity & $n_{o}$ & $n_{e}$ & $n_{s}$ \\
Unit & - & - & - \\
Value & 1.55 & 1.65 & 1.3
\end{tabular}




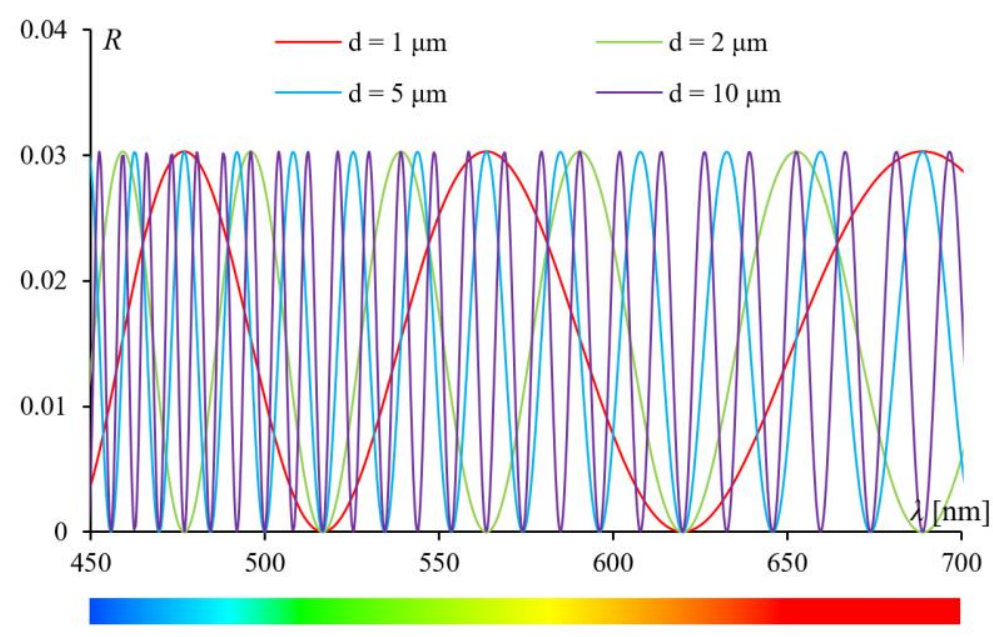

Figure 8: Reflectance spectra for different values of the thickness $d$.

(a)

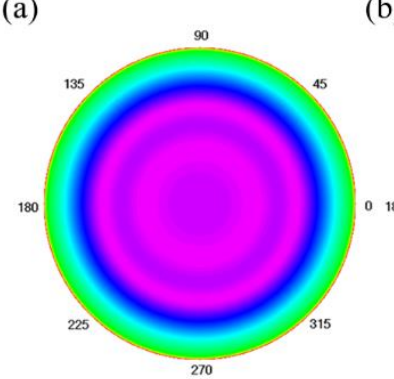

(b)

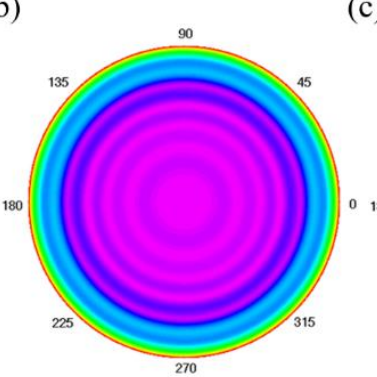

(c)

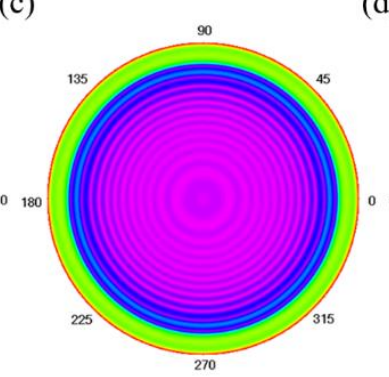

(d)

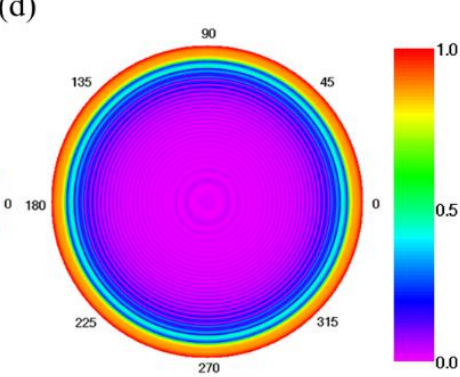

Figure 9: Polar plots of reflectance for different values of $d$ : (a) $d=1 \mu \mathrm{m}$; (b) $d=2 \mu \mathrm{m}$; (c) $d=5 \mu \mathrm{m}$; (d) $d=10 \mu \mathrm{m}$.

\subsection{Propagation of light in the helical structure of LC}

In the next step of the analysis, we presented some characteristics of the reflectance $R$ and the transmittance $T$ for different parameters of the CLC sandwiched within isotropic media. The parameters of the tested CLC are presented in Tab. 4. Figure 10 shows the reflectance and the transmittance spectra for normally incident non-polarized light in the whole visible wavelength range $380-780 \mathrm{~nm}$.

Table 4: Parameters of the investigated helical structure of $L C$ and isotropic optical medium.

\begin{tabular}{cccccc}
\hline Helical structure of $\mathbf{L C}\left(\phi_{c}(n)=2 \pi(d / p)(n / N), \theta_{c}(n)=90^{\circ}, n=1,2, \ldots, N\right.$ \\
\hline Quantity & $d$ & $p$ & $n_{o}$ & $n_{e}$ & $n_{s}$ \\
Unit & $\mu \mathrm{m}$ & $\mathrm{nm}$ & - & - & - \\
Value & 10 & 350 & 1.55 & 1.65 & 1.3
\end{tabular}

The obtained curve of the reflectance is characterized by the reflection bandwitch, i.e. the range of the wavelength of the incident light where $R=0.5$. The natural non-polarized incident light is the equal mixture of both right- and leftcircularly polarized light. Within the bandwidth, right-circularly polarized light is reflected by a right-handed helix, while left-circularly polarized light is transmitted by the cholesteric liquid crystal, and therefore values of both $R$ and $T$ coefficients are equal 0.5 . Concluding, we confirmed the occurrence of the selective reflection of the incident radiation within the bandwidth and transmission of both polarization states outside this bandwidth. 


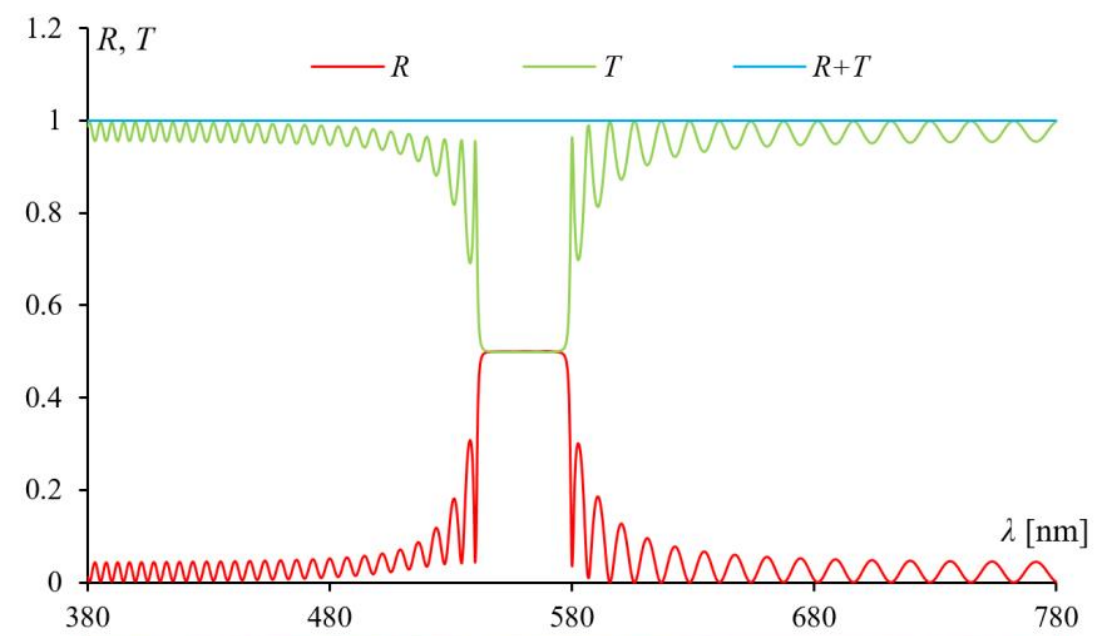

Figure 10: Reflectance and transmittance spectra of the normally incident non-polarized light.
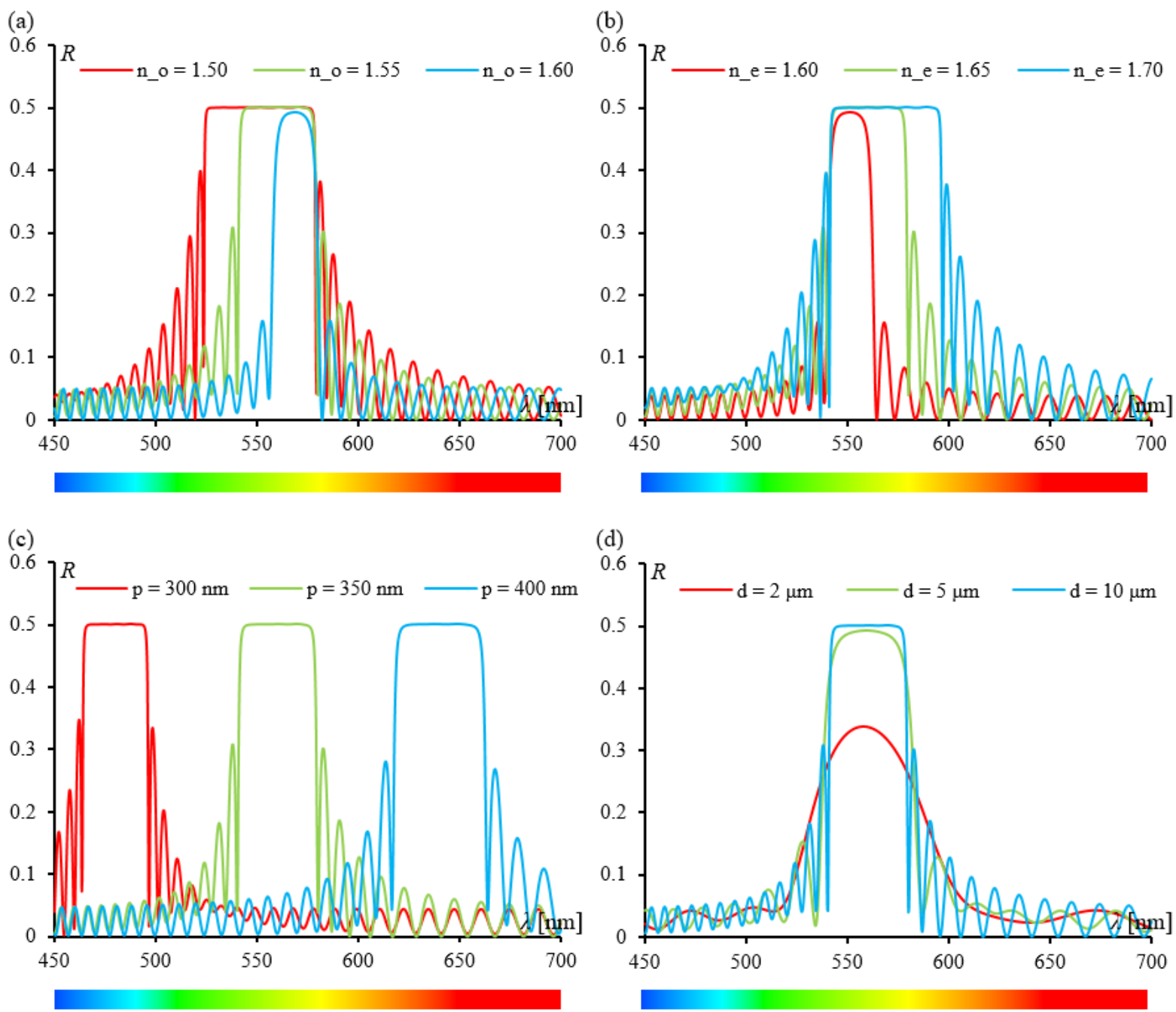

Figure 11: Reflection spectra of the normally incident non-polarized light: (a) for different values of $n_{o}$; (b) for different values of $n_{e} ;(c)$ for different values of $p$; (d) for different values of $d$.

Figure 11 presents reflection spectra for different parameters of the CLC. The calculations show that the bandwidth and its position in the wavelength spectrum strongly depends on the refractive indices $n_{o}$ and $n_{e}$ (see Figs. 11(a) and 
11(b)). The bandwidth also depends strongly on the pitch $p$ of the helical structure of the CLC (see Fig. 11(c)), whereas the shape of the bandwidth strongly depends on the thickness $d$ (see Fig. 11(d)).

In Figure 12(a), we presented the polar plot of the reflectance $R$ of the investigated CLC. The calculations were obtained for the wavelength $\lambda=560 \mathrm{~nm}\left(\lambda=p\left(n_{e}+n_{o}\right) / 2\right.$ - i.e. the middle of the selective reflection bandwidth presented in Fig. 10). As can be seen, the obtained plot does not depend on the angle $\phi$. However, we observed an interesting dependence on the angle $\theta$ presented in Figure 12(b), i.e. along the radius of the polar plot presented in Fig. 12(a). For small values of angle $\theta$, we obtained $T=0.5$ (selective reflection of the incident non-polarized light). For larger values of angle $\theta$, the reflectance $R$ decreases (due to mutual interferences in CLC both right- and left-circularly polarized light can be transmitted). For large values of angle $\theta$ (i.e. $\theta \approx 90^{\circ}$ ), the reflectance reaches 1 .

(a)

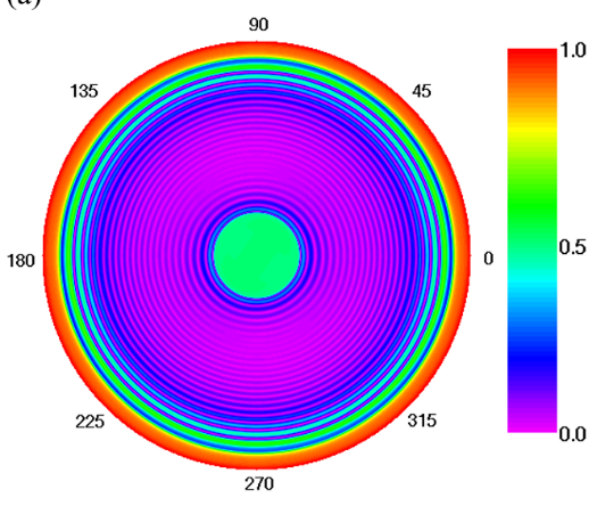

(b)

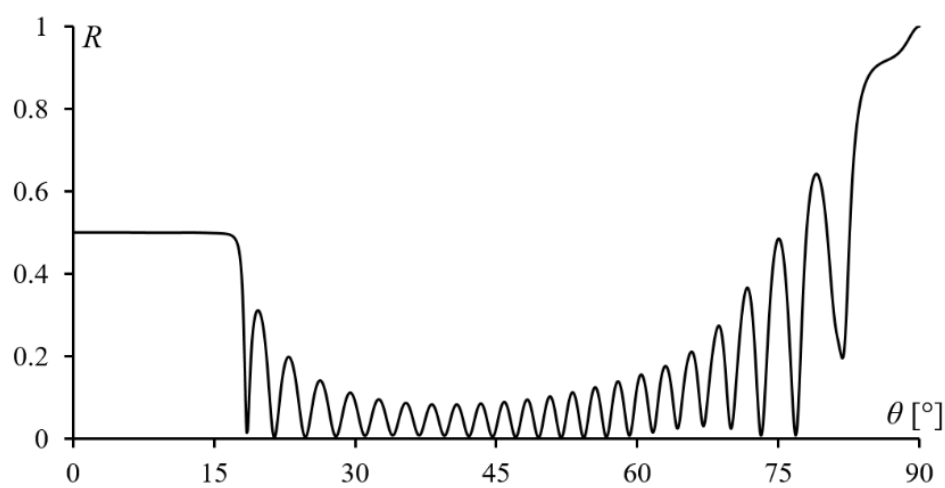

Figure 12: Polar plot (a) and the plot profile along the radius of the plot (b) of the reflectance.

Below we also presented interesting dependences of the reflection coefficient $R$ on the angle $\theta$ of the incident light. For all cases presented in Figs. 13-14, we took $\phi=0$, however, for thick layers of the CLC, this angle does not have a significant impact on the obtained dependences. Figure 13 shows the reflectance $R$ vs. the angle $\theta$ for different values of the wavelength $\lambda$ of the incidend light. As can be seen, for $\lambda=560 \mathrm{~nm}$, selective reflection occurs in the analysed system. For $\lambda=580 \mathrm{~nm}$, selective reflection does not occur, regardless of the angle $\theta$. In turn, for $\lambda=520 \mathrm{~nm}$ and $\lambda=540 \mathrm{~nm}$, we observed the selective reflection for the not normally incident light. In addition, in Figure 14, similar dependences, i.e., the reflection coefficient $R$ vs. the angle $\theta$, but for different values of the thickness $d$ of the investigated CLC, are presented. Both in Figs. 13 and 14, strong oscillations of the reflectance $R$ as a function of the angle $\theta$ are visible.

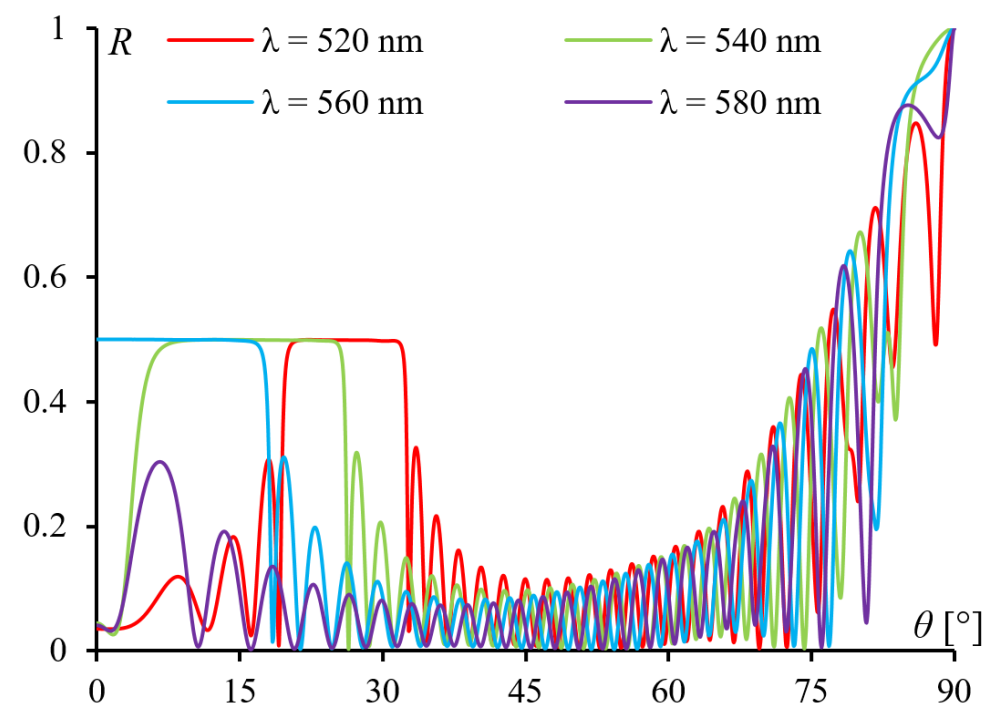

Figure 13: Reflectance vs. the angle $\theta$ for different values of the wavelength $\lambda$. 


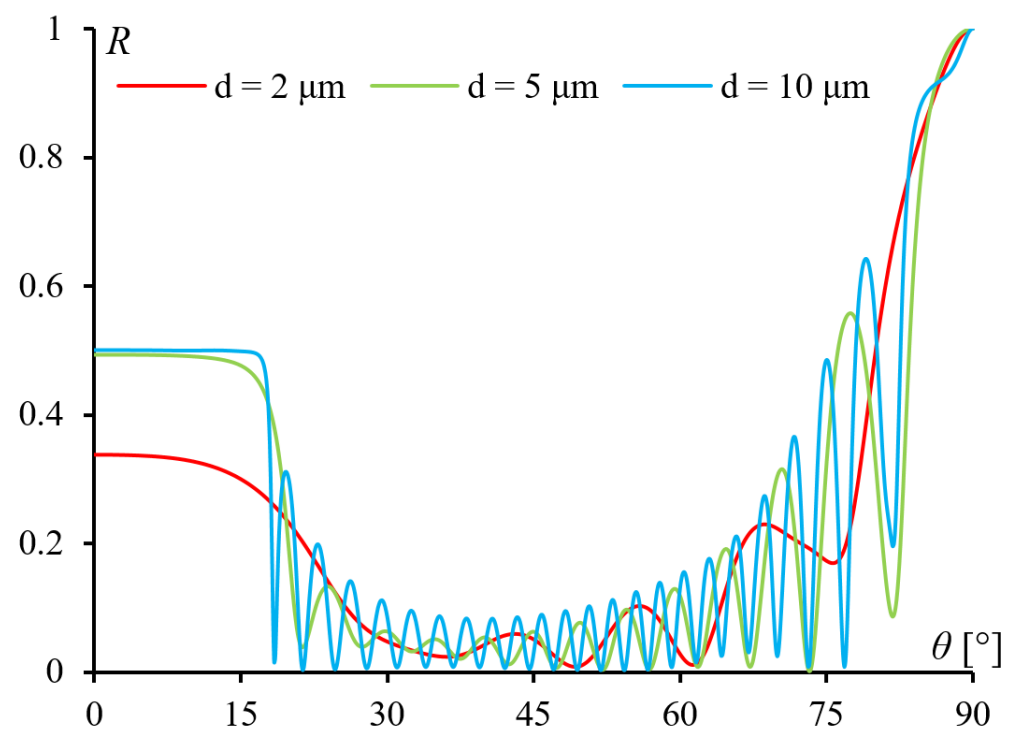

Figure 14: Reflectance vs. the angle $\theta$ for different values of the thickness $d$.

In Figs. 15-18, we presented polar plots of the reflectance of the investigated CLC for different values of the thickness $d$ and different wavelength $\lambda$ of the incident light. The obtained polar plots depend on both the thickness of the CLC layer and the wavelength of the incident light. For thin CLC layers, the reflectance $R$ oscillates slightly as a function of the angle $\theta$. In addition, the obtained distributions of the reflectance $R$ depend strongly on the angle $\phi$ of the incident light and the selective reflection phenomenon is not observed, even for $\lambda \approx p\left(n_{e}+n_{o}\right) / 2$. For thick layers of the CLC, we obtained symetrical distributions of the reflection coefficient $R$ and we detected selective reflection. Further analysis shows that for $\lambda=520 \mathrm{~nm}$ and $\lambda=540 \mathrm{~nm}$, the selective reflection occurs for the obligue (not normally) incident light. In turn, the widest range of the angle $\theta$, for which the selective reflection was observed, we obtained for $\lambda=545 \mathrm{~nm}$, i.e. $\lambda<p\left(n_{e}+n_{o}\right) / 2$ (see Fig. 17).

(a)

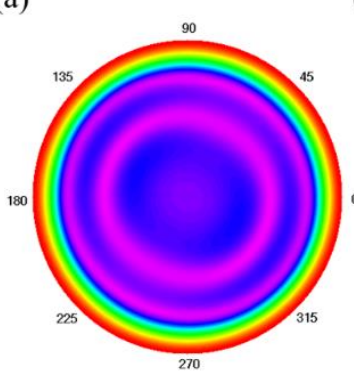

(b)

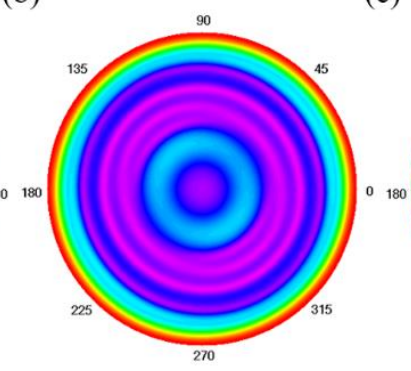

(c)

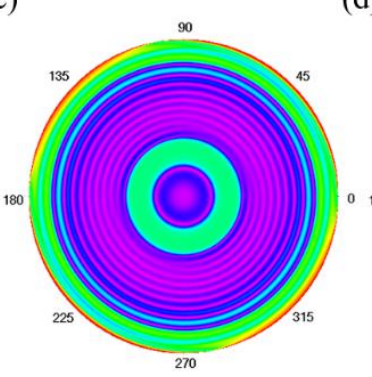

(d)

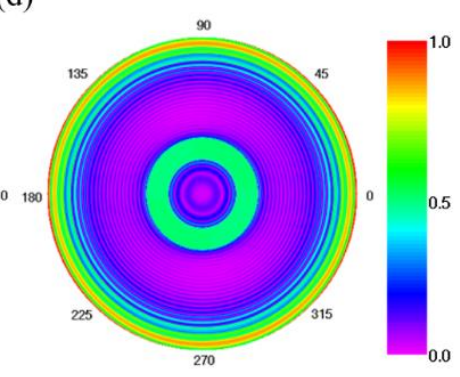

Figure 15: Polar plots of reflectance for $\lambda=520 \mathrm{~nm}$ and different values of the thickness $d$ : (a) $d=1 \mu \mathrm{m}$; (b) $d=2 \mu \mathrm{m}$; (c) $d=5 \mu \mathrm{m}$; (d) $d=10 \mu \mathrm{m}$.

(a)

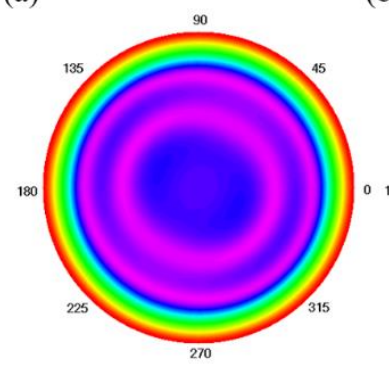

(b)

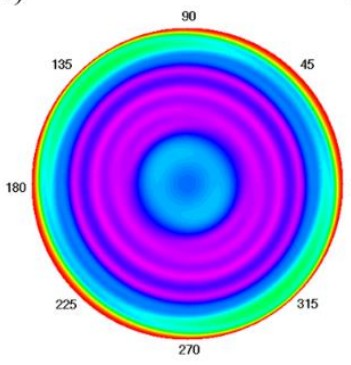

(c)

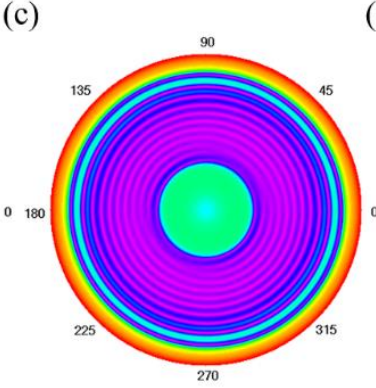

(d)

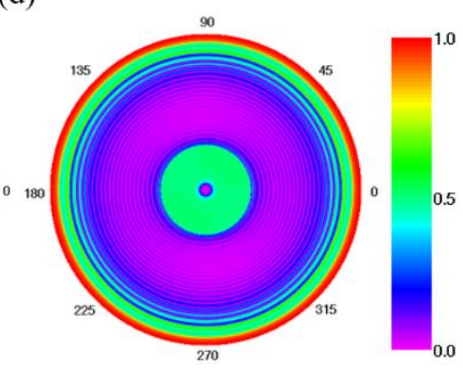

Figure 16: Polar plots of reflectance for $\lambda=540 \mathrm{~nm}$ and different values of the thickness $d$ :

(a) $d=1 \mu \mathrm{m}$; (b) $d=2 \mu \mathrm{m}$; (c) $d=5 \mu \mathrm{m}$; (d) $d=10 \mu \mathrm{m}$. 
(a)

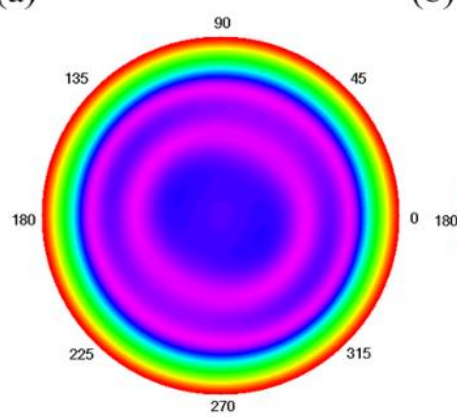

(b)

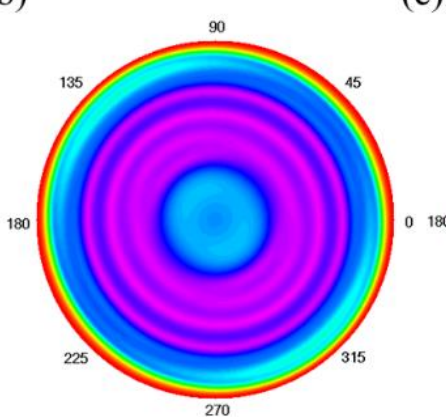

(c)

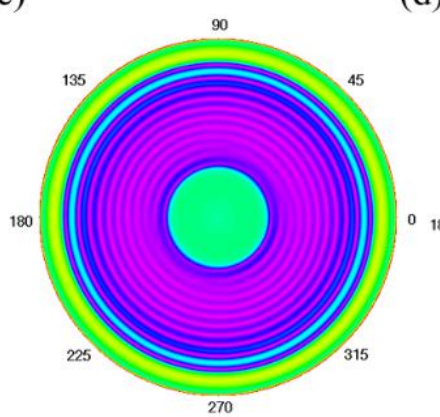

(d)

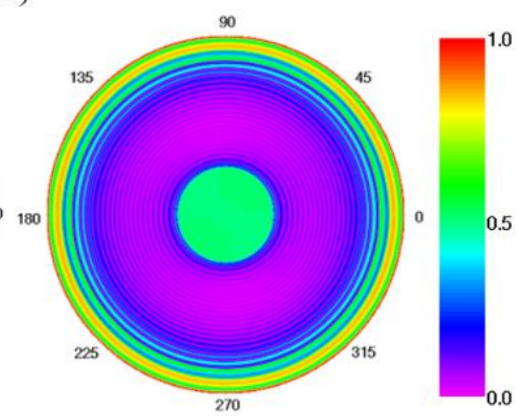

Figure 17: Polar plots of reflectance for $\lambda=545 \mathrm{~nm}$ and different values of the thickness $d$ : (a) $d=1 \mu \mathrm{m}$; (b) $d=2 \mu \mathrm{m}$; (c) $d=5 \mu \mathrm{m}$; (d) $d=10 \mu \mathrm{m}$.

(a)

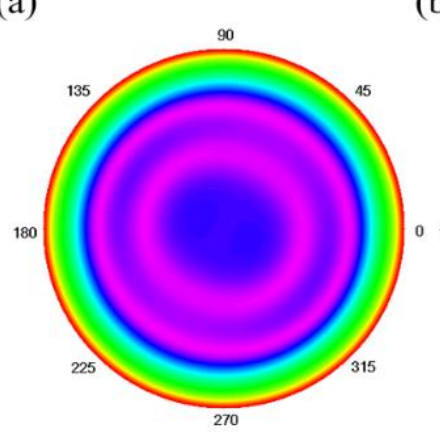

(b)

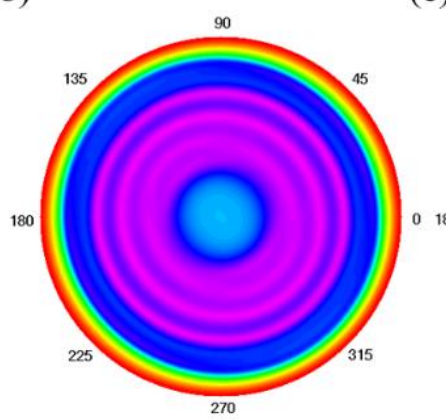

(c)

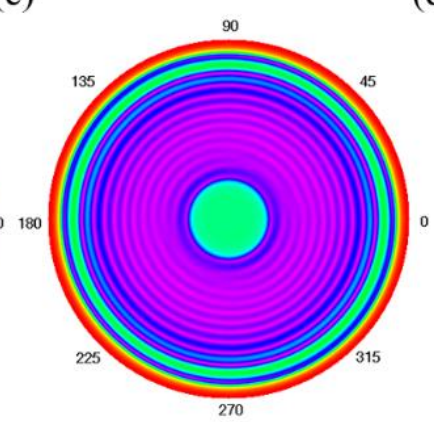

(d)

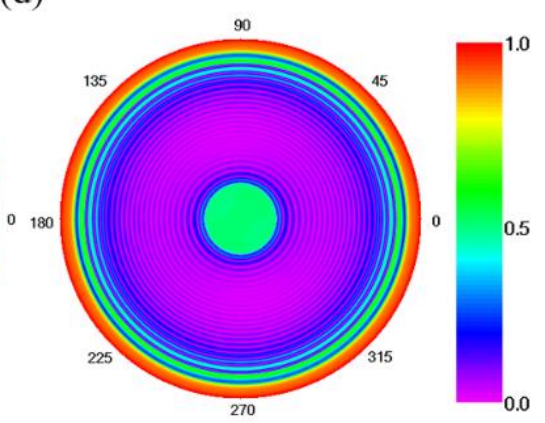

Figure 18: Polar plots of reflectance for $\lambda=560 \mathrm{~nm}$ and different values of the thickness $d$ :

(a) $d=1 \mu \mathrm{m}$; (b) $d=2 \mu \mathrm{m}$; (c) $d=5 \mu \mathrm{m}$; (d) $d=10 \mu \mathrm{m}$.

\section{CONCLUSIONS}

The paper attempts to model optical phenomena in different birefringent optical networks sandwiched between two isotropic media. To test the implemented algorithm, we considered the propagation of the light within homogenous isotropic medium and the propagation of the light in homogenous isotropic medium sandwiched within other isotropic medium first. Next, we calculated the reflection coefficient for the liquid crystals with different structures, especially cholesteric liquid crystals. Optical phenomena were modeled using the $4 \times 4$ matrix method, which takes into account the effect of refraction and multiple reflections between plate interferences, both ordinary and extraordinary waves that occur in birefringent networks. As a result, some interesting reflection and transmission spectra as well as polar plots of the reflection or transmission coefficient have been obtained, reported and discussed. The presented computer simulations allow to understand optical properties of the optical system analysed in this paper for different system parameters. And although the presented results have been obtained for birefringent networks with the positive dielectric anositropy (i.e. $n_{o}<n_{e}$ ), the implemented algorithm can be used for optical systems with negative dielectric anisotropy, i.e. $\left(n_{o}>n_{e}\right)$. In addition, assuming different distributions of the optical axis of the investigated liquid crystal, we are able to examine both reflection and transmittance spectra for the deformated LCs caused by the applied external electric field, external mechanical stresses or defects inside the crystal. These issues can be an interesting area for further research. Nevertheless, the results obtained in this paper can be adopted for understanding of optical phenomena in photonic crystals, which have attracted much attention in recent years. This is important from the engineering point of view, because they offer unique optical properties (including ability to control the propagation of light) and can be used as basic elements of all-optical integrated circuits in the future.

\section{Acknowledgments}

The work has been supported by the National Science Centre of Poland under the grant OPUS 14 no. 2017/27/B/ST8/01330 for years 2018-2021. 


\section{References}

Avendanno, C.G., Martinez, D., (2018). Elastic dependence of defect modes in one-dimensional photonic crystals with a cholesteric elastomer slab. Photonics and Nanostructures - Fundamentals and Applications 30:30-38.

Balamurugan, R., Liu, J.-H., (2016). A review of the fabrication of photonic band gap materials based on cholesteric liquid crystals. Reactive and Functional Polymers 105:9-34.

Belotti, M., Galisteo-López, J.F., De Angelis, S., Galli, M., Maksymov, I., Andreani, L.C., Peyrade, D., Chen Y., (2008). All-optical switching in 2D silicon photonic crystals with low loss waveguides and optical cavities. Optics Express 16:11624-11636.

Cardador, D., Vega, D., Segura, D., Trifonov, T., Rodriguez, A., (2017). Enhanced geometries of macroporous silicon photonic crystals for optical gas sensing applications. Photonics and Nanostructures - Fundamentals and Applications 25:46-51.

Chen, C.-J., Lien, A., Nathan, M.I., (1996). $4 \times 4$ matrix method for biaxial media and its application to liquid crystal displays. Japanese Journal of Applied Physics 35:L1204-L1207.

Chen, T., Feng, S.M., Xie, J.N., (2010). The new matrix and the polarization state of the transmitted light through the cholesteric liquid crystal. Optik 121:253-258.

Choi, S.S., Morris, S.M., Huck, W.T.S., Coles, H.J., (2009). Electrically tuneable liquid crystal photonic bandgaps. Advanced Materials 21:3915-3918.

Escobar, S., Nava, R., Flores-Romero, E., Reyes-Esqueda, J.A., (2018). Light polarization in active photonic waveguides of porous silicon. Photonics and Nanostructures - Fundamentals and Applications 31:44-51.

Fleming, J.G., Lin, S.Y., El-Kady, I., Biswas, R., Ho, K.M., (2002). All-metallic three-dimensional photonic crystals with a large infrared bandgap. Nature 417:52-55.

Gevorgyan, A.H., (2018). About defect mode peculiarities in cholesteric liquid crystals with anisotropic defect layer inside. Optik 154:656-661.

Gu, C., Yeh, P., (1999). Extended Jones matrix method and its application in the analysis of compensators for liquid crystal displays. Displays 20:237-257.

Guo, J., Wu, H., Chen, F., Zhang, L., He, W., Yang, H., Wei, J., (2010). Fabrication of multi-pitched photonic structure in cholesteric liquid crystals based on a polymer template with helical structure. Journal of Materials Chemistry 20:3997-4232.

Hart, E.E., Sobester, A., Djidjeli, K., Molinari, M., Thomas, K.S., Cox, S.J., (2012). A geometry optimization framework for photonic crystal design. Photonics and Nanostructures - Fundamentals and Applications 10:25-35.

Hirose, K., Liang, Y., Kurosaka, Y., Watanabe, A., Sugiyama, T., Noda, S., (2014). Watt-class high-power, high-beam-quality photonic-crystal lasers, Nature Photonics 8:406-411.

Hodgkinson, J., Tatam, R.P., (2013). Optical gas sensing: a review. Measurement Science and Technology 24:012004, 59 pages.

Huang, Y., Zhang, S., (2011). Optical filter with tunable wavelength and bandwidth based on cholesteric liquid crystals. Optics Letters 36:4563-4565.

Hussein, H.M.E., Ali, T.A., Rafat, N.H., (2018). A review on the techniques for building all-optical photonic crystal logic gates. Optics and Laser Technology 106:385-397.

Ivanov, O.V., Sementsov, D.I., (2000). Light propagation in stratified chiral media. The $4 \times 4$ matrix method. Crystallography Reports 45:487-492.

Kumar, R., Kumar, D., Kushwaha, A.S., Srivastava, S.K., (2018). Study of one-dimensional nanolayered graded photonic crystal consisting of birefringent and dielectric materials. Photonics and Nanostructures - Fundamentals and Applications 28:20-31.

Li, S.F., (2000). Jones-matrix analysis with Pauli matrices: Application to ellipsometry. Journal of the Optical Society of America A 17:920-926.

Lien, A., (1990a). The general and simplified Jones matrix representations for the high pretilt twisted nematic cell. Journal of Applied Physics 67:2853-2856.

Lien, A., (1990b). Extended Jones matrix representation for the twisted nematic liquid-crystal display at oblique incidence. Applied Physics Letters 57:2767-2769. 
Lin, T.-H., Li, Y., Wang, C.-T., Jau, H.-C., Chen, C.-W., Li, C.-C., Bisoyi, H.K., Bunning, T.J., Li, Q., (2013). Red, green and blue reflections enabled in an optically tunable self-organized 3D cubic nanostructures thin film. Advanced Materials 25:50505054.

Lin, J.-D., Chu, C.-L. Lin, H.-Y., You, B., Horng, C.-T., Huang, S.Y., Mo, T.-S., Huang, C.-Y., Lee, C.-R., (2015).Wide-band tunable photonic bandgaps based on nematic-refilling cholesteric liquid crystal polymer template samples. Optical Materials Express 5:1419-1430.

MacGregor, A.R., (1990). Method for computing homogeneous liquid-crystal conoscopic figures. Journal of the Optical Society of America A 7:337-347.

Mandal, P., (2018). Visible frequency plasmonic perfect absorber made of a thin metal layer containing cylindrical grooves. Photonics and Nanostructures - Fundamentals and Applications 31:66-70.

Morris, S.M., Hands, P.J.W., Findeisen-Tandel, S., Cole, R.H., Wilkinson, T.D., Coles, H.J., (2008). Polychromatic liquid crystal laser arrays towards display applications. Optics Express 16:18827-18837.

Nemati, H., Liu, S., Moheghi, A., Tondiglia, V.P., Lee, K.M., Bunning, T.J., Yang, D.-K., (2018). Enhanced reflection band broadening in polymer stabilized cholesteric liquid crystals with negative dielectric anisotropy. Journal of Molecular Liquids (https://doi.org/10.1016/j.molliq.2018.02.054).

Noda, S., (2010). Photonic crystal lasers-ultimate nanolasers and broad-area coherent lasers. Journal of the Optical Society of America B 27:B1-B8.

Notomi, M., (2010). Manipulating light with strongly modulated photonic crystals. Reports on Progress in Physics $73:$ id. 096501, 57 pages.

Nozaki, K., Kuramochi, E., Shinya, A., Notomi, M., (2014). 25-channel all-optical gate switches realized by integrating silicon photonic crystal nanocavities. Optics Express 22:14263-14274.

Ong, H.L., (1991a). Electro-optics of electrically controlled birefringence liquid-crystal displays by $2 \times 2$ propagation matrix and analytic expression at oblique angle. Applied Physics Letters 59:155-157.

Ong, H.L., (1991b). Electro-optics of a twisted nematic liquid-crystal display by $2 \times 2$ propagation matrix at oblique angle. Japanese Journal of Applied Physics Part 2 - Letters 30:L1028-L1031.

Ortega, J., Folcia, C.L., Etxebarria, J., (2018). Upgrading the performance of cholesteric liquid crystal lasers: Improvement margins and limitations. Materials 11:24 pages.

Ozaki, M., Matsuhisa, Y., Yoshida, H., Ozaki, R., Fujii, A., (2007). Photonic crystals based on chiral liquid crystal. Physica Status Solidi (a) 204: 3777-3789.

Prasmusinto, A., Sotto, M., Al-Attili, A.Z., Debnath, K., Saito, S., (2017). Theoretical designs for novel photonic crystal nanocavities with Si (111) interfaces. Photonics and Nanostructures - Fundamentals and Applications 26 (2017) 1-7.

Rudolph, P., (2016). Fundamentals and engineering of defects. Progress in Crystal Growth and Characterization of Materials 62:89-110.

Schwelb, O., (1986). Stratified lossy anisotropic media: general characteristics. Journal of the Optical Society of America A 3:188-193.

Tavousi, A., Heidarzadeh, H., (2018). Realization of a multichannel drop filter using an ISO-centric all-circular photonic crystal ring resonator. Photonics and Nanostructures - Fundamentals and Applications 31:52-59.

Wierer, J.J., David, A., Megens, M.M., (2009). III-nitride photonic-crystal light-emitting diodes with high extraction efficiency. Nature Photonics 3:163-169.

Wiesmann, C., Bergenek, K., Linder, N., Schwarz, U.T., (2009). Photonic crystal LEDs - designing light extraction. Laser \& Photonics Reviews 3:262-286.

Xu, H., Wu, P., Zhu, C., Elbaz, A., Gu, Z.Z., (2013). Photonic crystal for gas sensing. Journal of Materials Chemistry C 38:60876098.

Yang, O., Wang, Y., Wang, H., Yanling, H., Peng, J., (2015). Effect of stress-induced anisotropy on localized mode of onedimensional photonic crystal with mirror symmetry. Optik 126:5583-5586. 
Yeh, P., Gu, C. (1999). Optics of liquid crystal displays, John Wiley \& Sons, New York.

Zhang, J., Liu, H., Ding, Y., Wang, Y., (2018). A novel photonic crystal ring resonator configuration for add/drop filtering. Photonics and Nanostructures - Fundamentals and Applications 30:14-19. 